\title{
Electronic structure and exchange interaction in the layered perovskite $\mathrm{Sr}_{3} \mathrm{Mn}_{2} \mathrm{O}_{7}$
}

\author{
H. Meskine, Z. S. Popović, ${ }^{*}$ and S. Satpathy \\ Department of Physics \& Astronomy, University of Missouri, Columbia, Missouri 65211
}

(Received 23 May 2001; published 1 February 2002)

\begin{abstract}
The electronic structure of the Ruddlesden-Popper layered perovskite compound $\mathrm{Sr}_{3} \mathrm{Mn}_{2} \mathrm{O}_{7}$ is studied from density-functional calculations using the linear muffin-tin orbitals method. An antiferromagnetic, insulating solution is obtained in agreement with the experiments, with a magnetic moment of about $2.52 \mu_{B}$ for each Mn atom. The magnetic interactions between the Mn atoms, both within the bilayer and between the bilayers, are shown to arise from superexchange. The intrabilayer interaction involves the three-site Mn-O-Mn superexchange much like the case of the well-known $\mathrm{CaMnO}_{3}$, while the interbilayer exchange, mediated via the longer Mn-O-O-Mn superexchange path, is considerably weaker. Consistent with the layered nature of the compound, we find a strong out-of-plane to in-plane band-mass anisotropy for $\mathrm{Sr}_{3} \mathrm{Mn}_{2} \mathrm{O}_{7}\left(m_{z}^{*} / m_{x, y}^{*} \sim 10.9\right.$ for electrons and $\sim 4.2$ for holes), while for the related compound $\operatorname{LaSr}_{2} \mathrm{Mn}_{2} \mathrm{O}_{7}$, which is a ferromagnetic metal, we obtain a strong anisotropy in the resistivity $\rho_{c} / \rho_{a b} \sim 40$ using kinetic transport theory, in qualitative agreement with the experimental value of $\sim 100$.
\end{abstract}

DOI: 10.1103/PhysRevB.65.094402

PACS number(s): 75.10.Lp, 71.27.+a, 75.30.Vn

\section{INTRODUCTION}

The manganese-based perovskite oxides show a wide variety of novel magnetic field induced phenomena, such as the colossal magnetoresistance effect, spin, orbital, and charge ordering, melting of the charge order under the influence of the magnetic field, etc. ${ }^{1,2}$ Recently, considerable attention has been focused on a new series of manganites, viz., the $n=2$ members of the Ruddlesden-Popper compounds $\mathrm{La}_{2-2 x} \mathrm{Sr}_{1+2 x} \mathrm{Mn}_{2} \mathrm{O}_{7}(0 \leqslant x \leqslant 1)$, which comprise bilayers of $\mathrm{La}_{1-x} \mathrm{Sr}_{x} \mathrm{MnO}_{3}$ separated by insulating SrO layers (Fig. 1). ${ }^{3}$

Several members of the class exhibit large magnetoresistance and the two-dimensional nature of the compound is manifested in a number of magnetic and transport properties. For example, the two-dimensional character results in a striking anisotropy in resistivity. The presence of insulating $\mathrm{SrO}$ planes makes the resistivity $\rho_{c}$ normal to the planes to be two to three orders of magnitude greater than the resistivity $\rho_{a b}$ in the $a b$ plane. ${ }^{1}$ Another strong indication of the twodimensional character is that the measured critical exponent for the magnetization near $T_{c}$ is close to the theoretical exponent obtained from Onsager's exact solution of the twodimensional Ising model. ${ }^{4,5}$

In spite of the considerable current interest in these compounds, only a very few calculations of the electronic structure, mostly for the $x=0.5$ member of the series, have been reported. ${ }^{6,7}$ In this paper we focus our attention on the electronic and magnetic structures of the $x=1$ end member of the series, viz., $\mathrm{Sr}_{3} \mathrm{Mn}_{2} \mathrm{O}_{7}$, using density-functional methods and simple models for exchange and transport. The electronic structures of the end members are important as they provide considerable insight into the properties of the entire class of compounds and, with their smaller unit cells, have the simplest band structures. We note that $\mathrm{Sr}_{3} \mathrm{Mn}_{2} \mathrm{O}_{7}$ is analogous to the $x=1$ end member of the CMR perovskite series $\mathrm{La}_{1-x} \mathrm{Ca}_{x} \mathrm{MnO}_{3}$, with both having the nominal $\mathrm{Mn}$ valence of $\mathrm{Mn}^{4+}$, so that the $\mathrm{Mn} e_{g}$ states are empty. Both $\mathrm{Sr}_{3} \mathrm{Mn}_{2} \mathrm{O}_{7}$ and $\mathrm{CaMnO}_{3}$ are antiferromagnetic insulators.

The key results of our work may be summarized as fol- lows: (i) An insulating antiferromagnetic ground state is obtained within the local-density theory. (ii) The valence-band top and the conduction-band bottom both consist of bands with highly anisotropic masses, so that the charge carriers, introduced by either chemical doping or temperature, will lead to highly anisotropic conductivity within the relaxationtime approximation. (iii) The conductivity is much higher along the bilayer plane as compared to the direction normal to the plane, exactly as observed. An interesting result is that no significant in-plane anisotropy for resistivity along different directions in the plane is indicated from our results. (iv) The antiferromagnetic exchange interactions, both intrabilayer and interbilayer, are produced by superexchange, much like the familiar $\mathrm{CaMnO}_{3}$ case. Of these, the interbilayer exchange is found to be much smaller because of the longer superexchange path, which consists of four manganese and oxygen atoms. (v) For the $x=0.5$ compound of the series, viz., $\mathrm{LaSr}_{2} \mathrm{Mn}_{2} \mathrm{O}_{7}$, which is a ferromagnetic metal, we have also calculated the resistance anisotropy within the kinetic transport theory and, consistent with experiments, find a large anisotropy there as well.

\section{ELECTRONIC BAND STRUCTURE}

Several members of the layered perovskite family $\mathrm{SrO}\left(\mathrm{La}_{1-x} \mathrm{Sr}_{x} \mathrm{MnO}_{3}\right)_{2}$, including the end member $\mathrm{Sr}_{3} \mathrm{Mn}_{2} \mathrm{O}_{7}(x=1)$, crystallize in a tetragonal structure with the space group in the paramagnetic phase being $I 4 / \mathrm{mmm}$ (no. 139). ${ }^{8}$ This crystal structure is derived from the cubic perovskite structure and can be visualized as a stacking of sheets of double layers of $\mathrm{MnO}_{6}$ octahedra (Fig. 1). There are three types of oxygen atoms present in the structure: $\mathrm{O}(1)$ between two $\mathrm{Mn}$ atoms located on two different layers within the same bilayer, $\mathrm{O}(2)$ outside the bilayer forming the apex of the $\mathrm{MnO}_{6}$ octahedron, and $\mathrm{O}(3)$ located on the $\mathrm{MnO}_{2}$ layer, two of which form a bilayer. The three corresponding Mn-O bond lengths are 1.947, 1.901, and $1.895 \AA$ indicating the absence of any appreciable Jahn-Teller distortion, ${ }^{8}$ which is consistent with the expected $\mathrm{Mn}^{4+}$ nominal valence. However, in compounds where there is a mixture of $\mathrm{Mn}^{3+}$ and $\mathrm{Mn}^{4+}$, a significant octahedral distor- 


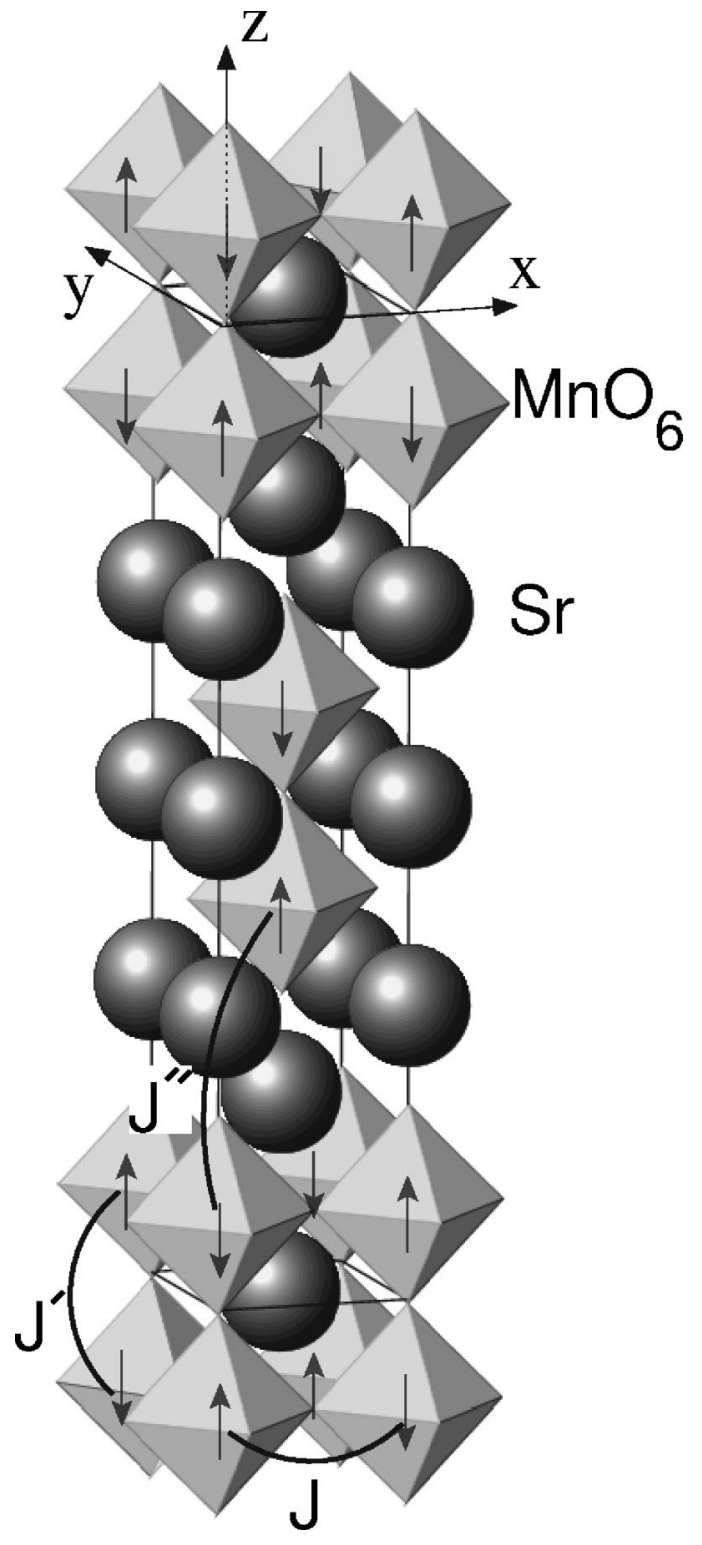

FIG. 1. Crystal structure of the bilayer perovskite $\mathrm{Sr}_{3} \mathrm{Mn}_{2} \mathrm{O}_{7}$. Arrows indicate the Mn magnetic moments. The intralayer, intrabilayer, and the interbilayer exchange interactions between the $\mathrm{Mn}$ atoms are denoted by $J, J^{\prime}$, and $J^{\prime \prime}$, respectively.

tion due to the Jahn-Teller interaction is found, resulting in large differences in the Mn-O bond lengths (e.g., 1.92 and $2.12 \AA$ in $\mathrm{La}_{1.4} \mathrm{Sr}_{1.6} \mathrm{Mn}_{2} \mathrm{O}_{7},{ }^{9}$ comparable to the distortion in $\mathrm{LaMnO}_{3}$ ).

The band-structure calculations presented here were performed using local spin-density approximation (LSDA) to the density-functional theory (DFT). The self-consistent tight-binding linear muffin-tin orbitals (TB LMTO) method was used. ${ }^{10}$ The structural data used in the electronic structure calculations are taken from Ref. 8. The atomic positions and the muffin-tin sphere radii are given in Table I. The magnetic unit cell is base-centered orthorhombic, space group $C 2 \mathrm{~mm}$ (no. 38 in the International Tables), with two formula units per unit cell. The calculations were scalar relativistic and the von Barth-Hedin ${ }^{11}$ exchange-correlation po-
TABLE I. Atom positions and sphere sizes for $\mathrm{Sr}_{3} \mathrm{Mn}_{2} \mathrm{O}_{7}$ used in the TB-LMTO calculation. Lattice parameters for the paramagnetic tetragonal unit cell (space group is $I 4 / \mathrm{mmm}$ ) are $a$ $=3.78936 \AA$ and $c=20.0638 \AA$ at $T=8 \mathrm{~K}$ (Ref. 8).

\begin{tabular}{lcccc}
\hline \hline Atom & $x / a$ & $y / a$ & $z / c$ & $\mathrm{~S}$ [a.u.] \\
\hline $\mathrm{Sr}(1)$ & 0.0 & 0.0 & \pm 0.5 & 3.73 \\
$\mathrm{Sr}(2)$ & 0.0 & 0.0 & \pm 0.31656 & 3.99 \\
$\mathrm{Mn}$ & 0.0 & 0.0 & \pm 0.09705 & 2.60 \\
$\mathrm{O}(1)$ & 0.0 & 0.0 & \pm 0.0 & 1.88 \\
$\mathrm{O}(2)$ & 0.0 & 0.0 & \pm 0.19181 & 1.60 \\
$\mathrm{O}(3)$ & 0.0 & 0.5 & \pm 0.09558 & 1.87 \\
\hline \hline
\end{tabular}

tential was used. Within the LMTO atomic sphere approximation (LMTO-ASA), the antiferromagnetic (AF) structure was found to be energetically favorable over the paramagnetic as well as the ferromagnetic structures.

The energy bands of $\mathrm{Sr}_{3} \mathrm{Mn}_{2} \mathrm{O}_{7}$ in the AF structure are presented in Fig. 2. An insulating solution is obtained, with an indirect gap of $0.45 \mathrm{eV}$ between the $\Gamma$ and the $Y$ points. The calculated magnetic moment is $\mu_{\mathrm{Mn}}=2.52 \mu_{B} / \mathrm{Mn}$, which is in good agreement with the measured value of $2.31 \mu_{B} / \mathrm{Mn}^{8}{ }^{8}$ According to the Hund's rule the magnetic moment for the free $\mathrm{Mn}^{4+}$ ion should be $3 \mu_{B} / \mathrm{Mn}$, which is reduced by the hybridization effects in the crystal. The magnetic moment is carried almost entirely by the $t_{2 g}$ electrons,

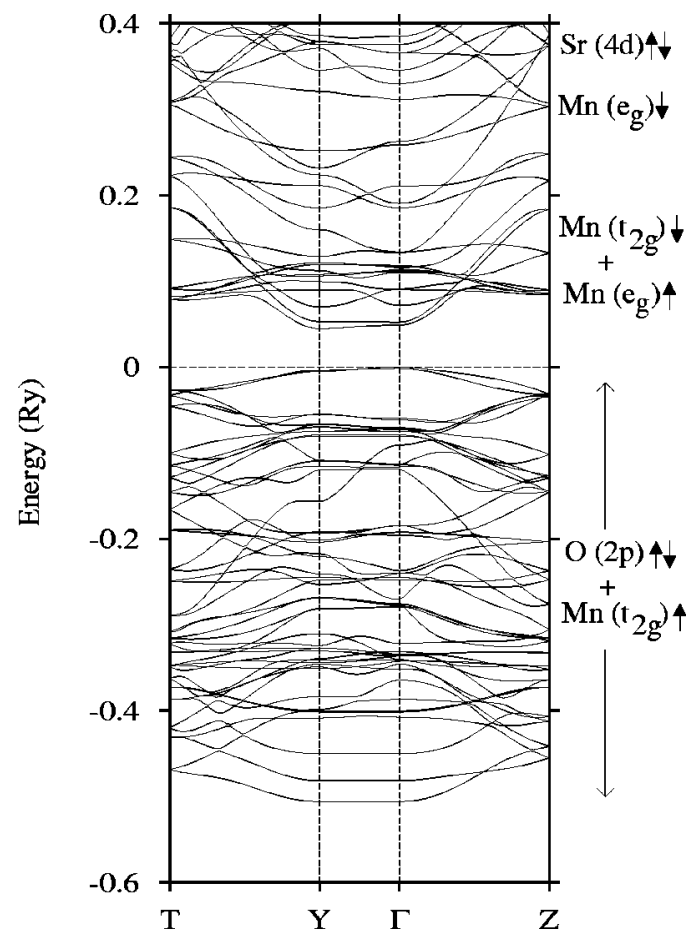

FIG. 2. Density-functional band structure for $\mathrm{Sr}_{3} \mathrm{Mn}_{2} \mathrm{O}_{7}$ with the antiferromagnetic crystal structure. The valence- and conduction-band dispersions in the gap region are strongly anisotropic, which leads in turn to a highly anisotropic conductivity for the doped carriers as discussed in the text. The $\vec{k}$ points are: $\Gamma$ $=(0,0,0), Y=(0,0, a / c), Z=(1 / 4,1 / 4,0)$, and $T=(1 / 4,1 / 4, a / c)$, in units of $2 \pi / a$. 


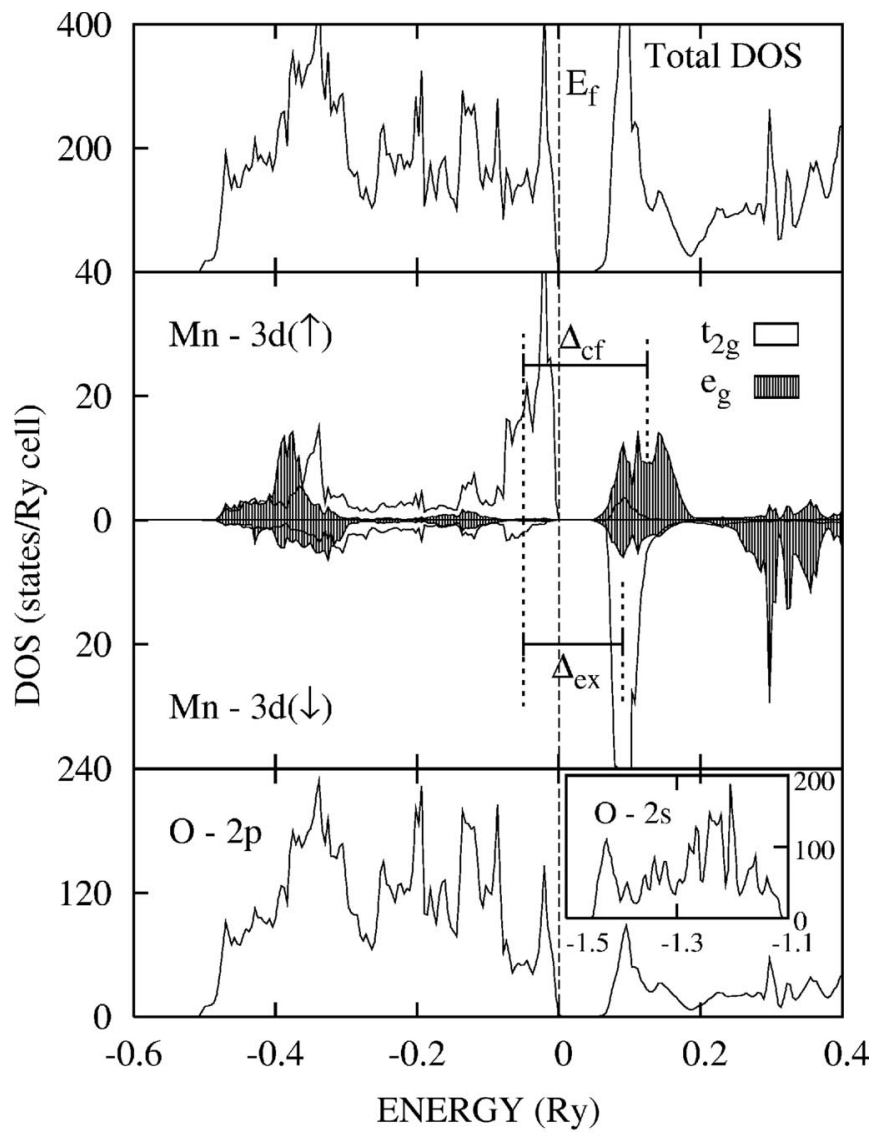

FIG. 3. Density of states for $\mathrm{Sr}_{3} \mathrm{Mn}_{2} \mathrm{O}_{7}$. The valence bands are of combined $\mathrm{O}(p)-\mathrm{Mn}\left(t_{2 g} \uparrow\right)$ character, while the conduction bands near the gap consist primarily of $\operatorname{Mn}\left(t_{2 g} \downarrow\right)$ and $\operatorname{Mn}\left(e_{g} \uparrow\right)$ states. Symbols $\Delta_{c f}$ and $\Delta_{e x}$ indicate, respectively, the crystal-field splitting and the exchange splitting.

causing the $t_{2 g}$ bands to be completely spin polarized.

The bands are consistent with the nominal chemical valence of $\mathrm{Mn}^{4+}\left(t_{2 g}^{3} e_{g}^{0}\right)$. Note that the chemical valence of the series may be represented as $\left(\mathrm{La}_{1-x}^{3+} \mathrm{Sr}_{x}^{2+} \mathrm{Mn}^{(3+x)+} \mathrm{O}_{3}^{2-}\right)_{2}\left(\mathrm{Sr}^{2+} \mathrm{O}^{2-}\right)$, where the first parantheses indicates the bilayer and the second, the insulating $\mathrm{SrO}$ layer. An electronic configuration with empty $e_{g}$ orbitals implies a Jahn-Teller inactive system. The Mn valence being $\mathrm{Mn}^{4+}$, the electronic structure has considerable similarity with that of $\mathrm{CaMnO}_{3} .{ }^{12,13}$ The Mn-3d states are split into a triply degenerate $t_{2 g}$ and a doubly degenerate $e_{g}$ state by the cubic crystal field, as seen from the projected density of states (DOS) shown in Fig. 3. The crystal-field splitting of $\sim 2 \mathrm{eV}$ is comparable to the strength of the exchange splitting $(\sim 2 \mathrm{eV})$, putting the $t_{2 g}^{\downarrow}$ and the $e_{g}^{\uparrow}$ states in the same energy region. In the layered systems, the symmetry of the crystal field is reduced from cubic to tetragonal, which would remove the degeneracies of the $t_{2 g}$ and the $e_{g}$ states. However, the present compound being Jahn-Teller inactive, this effect is small. The characteristic features of the present DOS curves are essentially similar to those for the cubic perovskite $\mathrm{CaMnO}_{3}$.

The valence electron charge-density contours, shown in Fig. 4 , are consistent with the occupation of the oxygen $2 p$

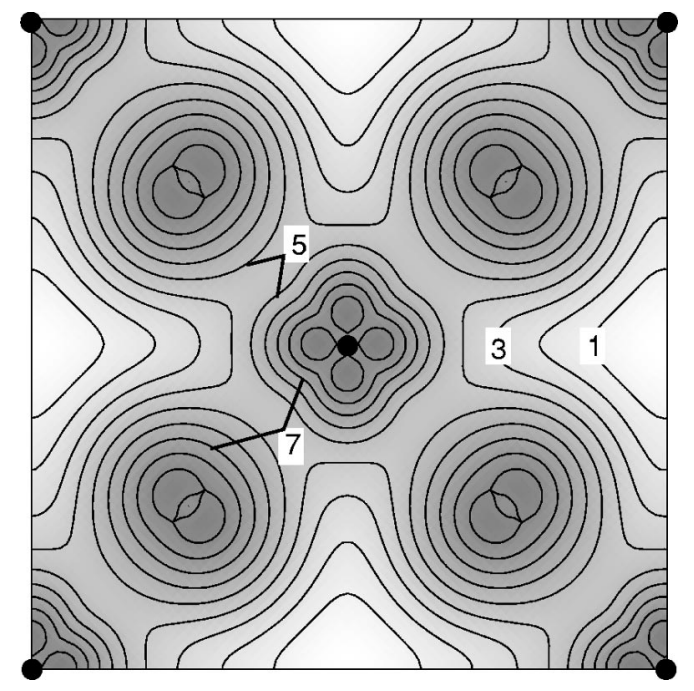

FIG. 4. Valence electron charge-density contours plotted on the $\mathrm{MnO}_{2}$ layer indicating the bonding interaction between the occupied $\operatorname{Mn}\left(t_{2 g}\right)$ and the oxygen $p$ orbitals. Contour values are: $\rho_{n}$ $=\rho_{0} \times 10^{-n \delta} e^{-} / a_{0}^{3}$, where $\rho_{0}=4.7 \times 10^{-3}, \delta=0.47, a_{0}$ is the Bohr radius, and $n$ labels the contours.

shell and the Mn $\left(t_{2 g}\right)$ states. The lobes on the atoms seen in the figure are indicative of the bonding interaction between the atoms.

\section{RESISTIVITY ANISOTROPY}

\section{A. $\mathrm{Sr}_{3} \mathrm{Mn}_{2} \mathrm{O}_{7}$}

The band structure shows a large anisotropy in the dispersion of both the valence and the conduction bands in the gap region. This would lead to a large anisotropy in the in-plane vs out-of-plane conductivity in samples with carriers, introduced either by chemical doping or by finite temperature. We have estimated this anisotropy ratio for $\mathrm{Sr}_{3} \mathrm{Mn}_{2} \mathrm{O}_{7}$ within the simple relaxation-time approximation (RTA) where the conductivity tensor may be written as $\bar{\sigma}=n|e| \bar{\mu}$, where $n$ is the concentration of the carriers, $e$ is their charge, and $\bar{\mu}$ is the carrier mobility. ${ }^{14}$ The mobility is given by $\bar{\mu}=|e| \tau M^{-1}$ where $\tau$ is the average relaxation time and $M$ is the effectivemass tensor, $M^{-1}=\left(1 / \hbar^{2}\right) \partial^{2} E(\vec{k}) / \partial \vec{k}^{2}$. The energies of the valence and the conduction bands may be written as $E(\vec{k})$ $=E_{c, v} \pm \hbar^{2}\left(k_{x}^{2} / 2 m_{x}^{*}+k_{y}^{2} / 2 m_{y}^{*}+k_{z}^{2} / 2 m_{z}^{*}\right)$, where $E_{c}\left(E_{v}\right)$ is the bottom (top) of the conduction (valence) band and $m_{x}^{*}$, $m_{y}^{*}$, and $m_{z}^{*}$ are the principal values of the effective masses obtained by diagonalizing the mass tensor. In the RTA, the conductivity along different crystallographic directions is then determined simply by the mass anisotropy.

We have estimated the ratio of the principal values of the masses by examining the band dispersion along different directions in the crystal. Figure 2 already indicates that the band dispersion is rather flat along directions perpendicular to the bilayers $(\vec{k}$ along $\Gamma-Y)$ for both the valence top and the conduction bottom. For the conduction bottom, the ratio of the band masses may be obtained from Fig. 5, which shows a contour plot of the band energies. An estimate of the 


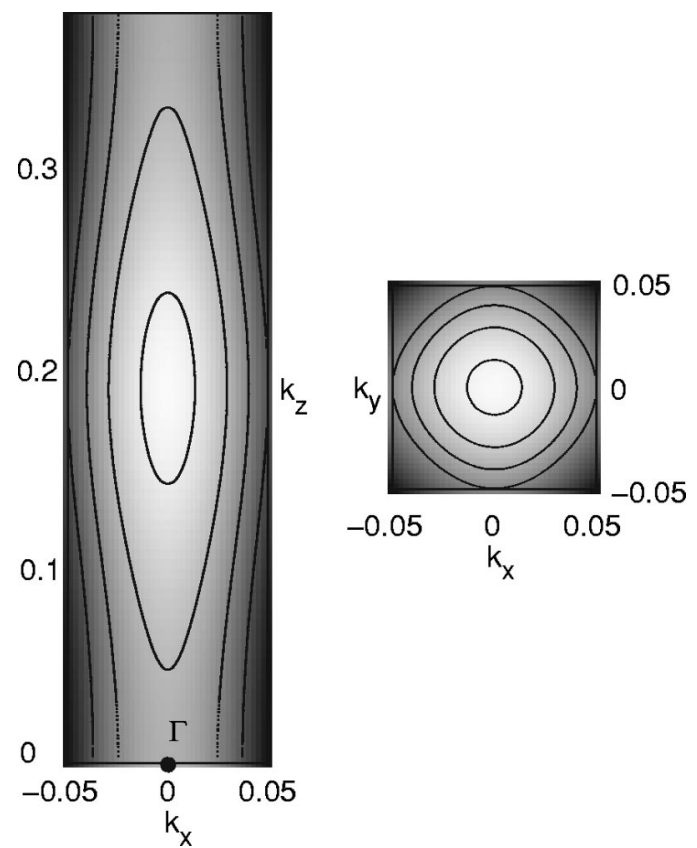

FIG. 5. Constant energy contours near the conduction bottom indicating the highly anisotropic in-plane vs out-of-plane band dispersion. The coordinate system for $\vec{k}$ is the same as in Fig. 1 and contours on both plots have the same values. The plot on the right part indicates isotropic dispersion along different directions on the bilayer plane. Bloch momentums are in units of $2 \pi / a$.

in-plane to out-of-plane conductivity ratio for the electrons is then found to be $\sigma_{a b} / \sigma_{c} \approx m_{z}^{*} / m_{x, y}^{*} \sim 10.9$. Note also from the contours that the band mass is isotropic along the plane of the bilayers. Similarly, the anisotropy in the hole conductivity was found to be $\sigma_{a b} / \sigma_{c} \approx m_{z}^{*} / m_{x, y}^{*} \sim 4.2$.

To our knowledge, although $\mathrm{Sr}_{3} \mathrm{Mn}_{2} \mathrm{O}_{7}$ is known to be an insulator, there is no quantitative measurement of the resistivity anisotropy of either doped samples or undoped samples at finite temperatures. However, measurements have been made for the related bilayer compound $\mathrm{LaSr}_{2} \mathrm{Mn}_{2} \mathrm{O}_{7}{ }^{15}$ which is the $x=0.5$ member of the series and is a ferromagnetic metal. We have computed the anisotropy for this compound as well, results of which are described below.

\section{B. $\mathrm{LaSr}_{2} \mathrm{Mn}_{2} \mathrm{O}_{7}$}

A rough estimate for the resistivity anisotropy for the metallic $\mathrm{LaSr}_{2} \mathrm{Mn}_{2} \mathrm{O}_{7}$ is obtained using band dispersions at the Fermi energy and kinetic transport theory. According to the latter, the conductivity is given by $\sigma=\frac{1}{3} \tau e\left\langle v_{f}^{2}\right\rangle N_{f}$, where $\tau$ is an average relaxation time, $N_{f}$ is the Fermi energy electron density, and $v_{f}$ is the Fermi velocity.

To estimate the quantities entering into the kinetic transport expression, we have performed a band calculation for the $\mathrm{LaSr}_{2} \mathrm{Mn}_{2} \mathrm{O}_{7}$ using the full-potential LMTO method. ${ }^{16}$ We used a double- $\kappa$ basis set for the $s, p$, and $d$ muffin-tin orbitals, with their tails expanded about other sites in spherical harmonics up to $l_{\max }=6$. The calculation was performed with the Perdew-Wang exchange-correlation potential in the generalized gradient approximation (GGA). ${ }^{17}$ The calculated

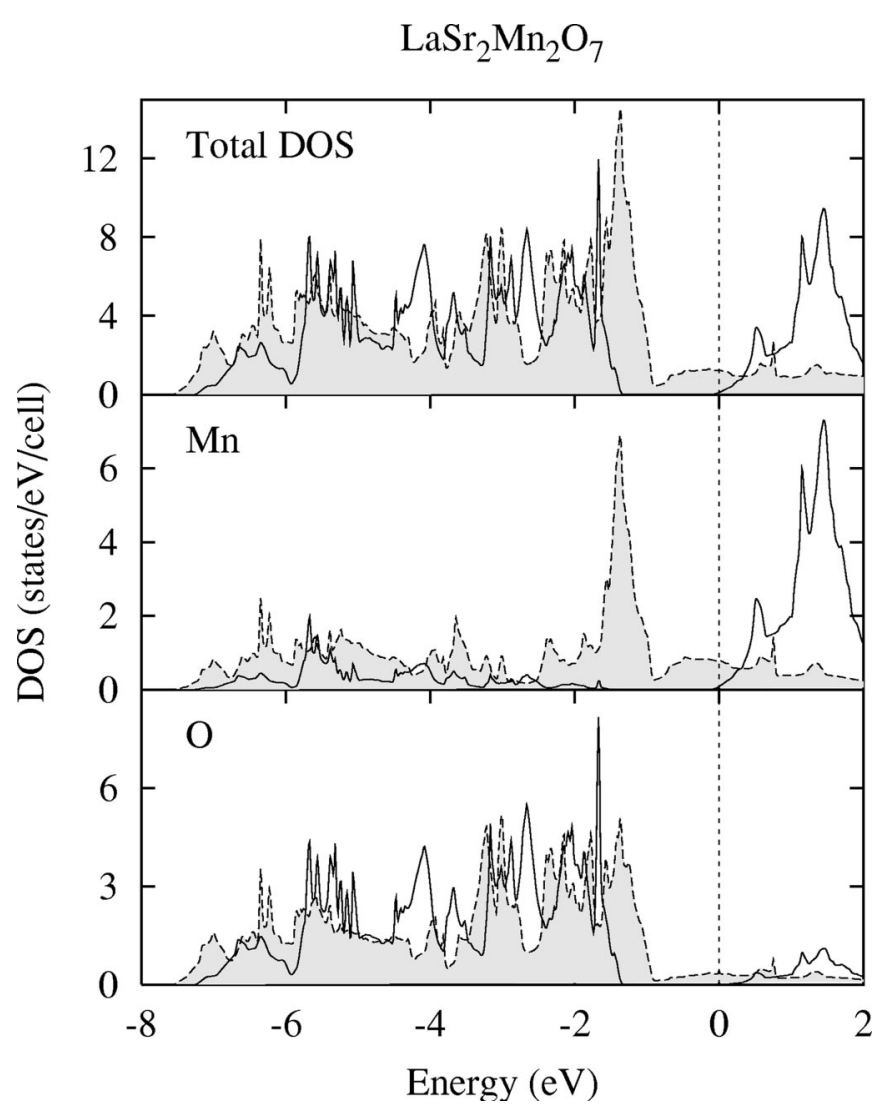

FIG. 6. Densities of states for the ferromagnetic $\mathrm{LaSr}_{2} \mathrm{Mn}_{2} \mathrm{O}_{7}$, calculated using the full-potential LMTO method and Perdew-Wang exchange-correlation in the GGA. Results indicate a nearly half metallic system. Densities of states corresponding to the majority spins are indicated by solid lines while those corresponding to the minority spins are indicated by dashed lines.

densities of states are shown in Fig. 6. We find a nearly half metallic band structure for this compound in agreement with the earlier results of de Boer and de Groot. ${ }^{6}$ A small density of states at the Fermi energy is found for the minority electrons allowing conduction in both the majority and the minority spin channels. If the local-density approximation (LDA) is used instead of the GGA in the calculation, the minority conduction bands shift slightly down thereby increasing the minority DOS at the Fermi energy.

Even though both LDA and GGA indicate the presence of a small number of minority electrons at the Fermi energy, we find that they contribute very little to the conductivity because of their comparatively small Fermi velocity. Our GGA calculation for $\mathrm{LaSr}_{2} \mathrm{Mn}_{2} \mathrm{O}_{7}$ yields the following values for the DOS at the Fermi level: $N_{\uparrow(\downarrow)}\left(E_{f}\right)=16.67$ (1.64) states/ Ry/unit cell, while the averaged Fermi velocities for spin majority (minority) are: $\left\langle v_{x}^{2}\right\rangle_{\uparrow(\downarrow)}=\left\langle v_{y}^{2}\right\rangle_{\uparrow(\downarrow)}=2.70$ $(0.09) \times 10^{12} \mathrm{~m} / \mathrm{s},\left\langle v_{z}^{2}\right\rangle_{\uparrow(\downarrow)}=0.14 \quad(0.0) \times 10^{12} \mathrm{~m} / \mathrm{s}$. Notice that not only are the minority-spin electrons smaller in number, but also that they have a much smaller Fermi velocity (heavier effective mass), making the majority-spin electrons responsible for the bulk of the conductivity. The Fermi velocities are also strongly anisotropic, being much smaller in the out-of-plane direction, leading to a strong anisotropy in 

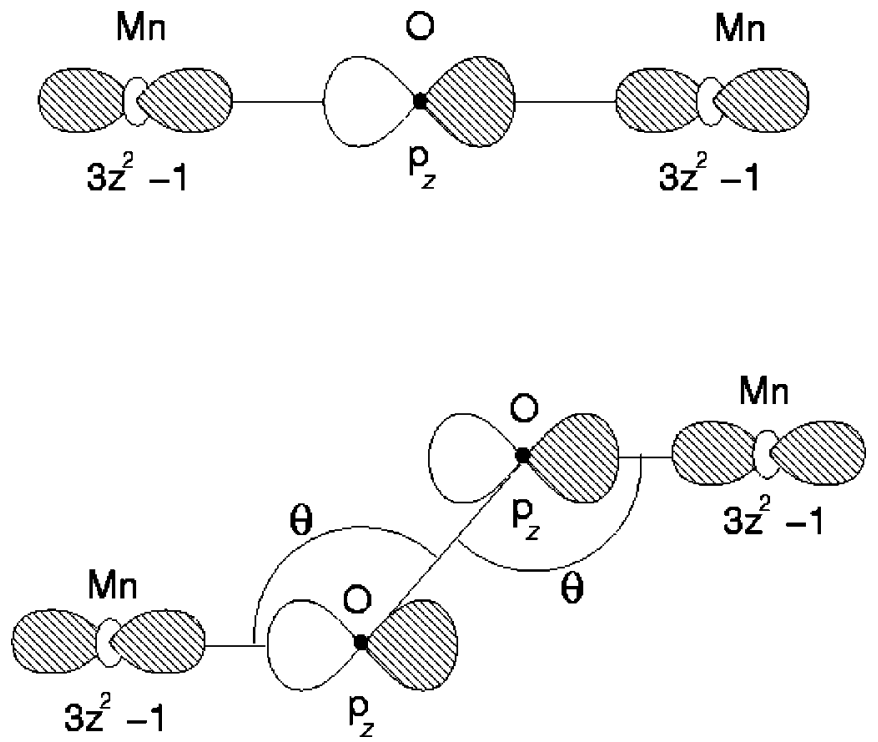

FIG. 7. Superexchange paths for the intralayer and intrabilayer exchange (a) and for the interbilayer exchange (b). Defining the $z$ axis along the $\mathrm{Mn}-\mathrm{O}$ bonds, only the electron hopping involving the $\mathrm{O}\left(p_{z}\right)$ and the $\operatorname{Mn}\left(z^{2}-1\right)$ orbitals contributes to the magnetic exchange in the lowest order of the perturbation theory. The Mn-O-O angle $\theta$ is about $135^{\circ}$ for $\mathrm{Sr}_{3} \mathrm{Mn}_{2} \mathrm{O}_{7}$, while the $\mathrm{Mn}-\mathrm{O}-\mathrm{Mn}$ bond is close to $180^{\circ}$.

resistivity $\rho_{c} / \rho_{a b}=\sigma_{a b} / \sigma_{c} \sim 40$ in qualitative agreement with the experimental value of $\sim 100$. $^{15}$

\section{EXCHANGE INTERACTION}

The magnetic structure of $\mathrm{Sr}_{3} \mathrm{Mn}_{2} \mathrm{O}_{7}$ is shown in Fig. 1 The exchange interactions can be explained within a superexchange model involving either the triatomic $\mathrm{Mn}-\mathrm{O}-\mathrm{Mn}$ path for the intralayer and the intrabilayer exchanges or the tetratomic Mn-O-O-Mn path for the interbilayer exchange. Throughout this paper, we denote the intralayer, intrabilayer, and the interbilayer exchange interactions between the Mn atoms by $J, J^{\prime}$, and $J^{\prime \prime}$, respectively (see Fig. 1).

To describe the exchange, we consider the following Hamiltonian model involving the $\operatorname{Mn}(d)$ and $\mathrm{O}(p)$ electrons, with the $t_{2 g}$ spins treated as core:

$$
\begin{aligned}
H= & \sum_{i \alpha} \epsilon_{i \alpha} n_{i \alpha}+\sum_{i \alpha, j \beta \sigma} t_{i \alpha j \beta}\left(c_{i \alpha \sigma}^{\dagger} c_{j \beta \sigma}+\text { H.c. }\right) \\
& +U_{d} \sum_{i} n_{i}\left(n_{i}-1\right)-J_{H} \sum_{i} \vec{S}_{i} \cdot \vec{\sigma}_{i} .
\end{aligned}
$$

Here $i, \alpha$, and $\sigma$ are, respectively, the site, orbital and spin indices, $c_{i \alpha \sigma}^{\dagger}$ is the corresponding electron creation operator, $\epsilon_{i \alpha}$ 's are the on-site energies, $U_{d}$ is the Coulomb energy on the Mn site, and $J_{H}$ is the Hund's rule exchange energy. We shall, for simplicity, take $J_{H}=\infty$ so that only the $e_{g}$ states parallel to the $t_{2 g}$ spin at a Mn site can be occupied. The $t_{2 g}$ electrons are treated as classical core spins denoted by $\vec{S}_{i}$ at the $i$ th site. Hopping is allowed between $\operatorname{Mn}\left(e_{g}\right)$ and $\mathrm{O}(p)$ orbitals at the nearest-neighbor sites.
If now the $z$ axis in the local coordinate system is chosen along the Mn-O bond (see Fig. 7), then it is easy to see that the only important contribution to the exchange comes from electron hopping involving the $\operatorname{Mn}\left(z^{2}-1\right)$ and the $\mathrm{O}\left(p_{z}\right)$ orbitals. This is because the intralayer and the intrabilayer exchanges involve the linear $\mathrm{Mn}-\mathrm{O}-\mathrm{Mn}$ triad with a roughly $180^{\circ} \mathrm{Mn}-\mathrm{O}-\mathrm{Mn}$ bond angle, which makes the hopping integral involving the $\mathrm{O}\left(p_{x}\right)$ and $\mathrm{O}\left(p_{y}\right)$ orbitals zero by symmetry. As for the interbilayer exchange $J^{\prime \prime}$, although the $\mathrm{O}\left(p_{x}\right)$ and $\mathrm{O}\left(p_{y}\right)$ orbitals are coupled to the system on account of the deviation of the Mn-O-Mn angle from a straight bond, these hoppings contribute to the exchange in higher orders of the perturbation theory. The interatomic hopping integrals are described through the use of the Koster-Slater tightbinding matrix elements.

The exchange interaction $J$ between two Mn atoms is obtained by calculating the ground-state energies corresponding to the ferromagnetic (FM) and antiferromagnetic (AF) alignment of the two $t_{2 g}$ spins and then taking the difference:

$$
J_{e x}=E_{\uparrow \uparrow}-E_{\uparrow \downarrow},
$$

where positive (negative) values of $J_{e x}$ indicate an AF (FM) interaction between the $t_{2 g}$ spins. Note that our $J_{e x}$ is related to the Heisenberg exchange parameter $\bar{J}$ appearing in the standard expression for the Heisenberg Hamiltonian $H=-\bar{J} \Sigma_{i j} \vec{S}_{i} \cdot \vec{S}_{j}$ via the equation $J_{e x}=4 \bar{J} S^{2}$.

\section{A. Intralayer and intrabilayer exchanges: $\mathrm{Mn}-\mathrm{O}-\mathrm{Mn}$ case}

Consider first the intralayer and the intrabilayer magnetic exchanges, $J$ and $J^{\prime}$ (Fig. 1), which are mediated between two $\mathrm{Mn}$ atoms via $\mathrm{O}(3)$ and $\mathrm{O}(1)$, respectively. There are just two relevant parameters in the Hamiltonian, viz., the oxygen to manganese charge-transfer energy $\Delta \equiv E\left(d^{4} p^{5}\right)$ $-E\left(d^{3} p^{6}\right) \approx 5 \mathrm{eV}$ and the Mn-O $t_{p d \sigma}$ hopping matrix element which we denote by $t \approx-1 \mathrm{eV}$.

Straight $\mathrm{Mn}-\mathrm{O}-\mathrm{Mn}$ bond. For a straight bond, since the active orbitals (nonzero hopping) are just the $\mathrm{O}\left(p_{z}\right)$ and the $\operatorname{Mn}\left(z^{2}-1\right)$ orbitals, in the FM case we have two $\mathrm{O}\left(p_{z}\right)$ electrons, taking both spins into account. There are three spin-up orbitals and one spin-down orbital available for these electrons, resulting in a three-dimensional configuration space $\left({ }^{3} C_{1} \times{ }^{1} C_{1}\right)$. The basis set is explicitly: $|100\rangle,|010\rangle$, and $|001\rangle$, corresponding to the lone spin-up electron occupying either the $\mathrm{O}(p)$ or one of the two $\operatorname{Mn}(d)$ orbitals. The spindown electron can only be on the $\mathrm{O}(p)$ orbital in the FM case due to the infinite $J_{H}$. The Hamiltonian is then simply the $3 \times 3$ matrix:

$$
H_{\uparrow \uparrow}=\left(\begin{array}{ccc}
0 & t & t \\
t & \Delta & 0 \\
t & 0 & \Delta
\end{array}\right) .
$$

The ground-state energy is then given by

$$
E_{\uparrow \uparrow}=\left(\Delta-\sqrt{\Delta^{2}+8 t^{2}}\right) / 2 .
$$

For the AF of the Mn spins, we obtain the following Hamiltonian: 


$$
H_{\uparrow \downarrow}=\left(\begin{array}{cccc}
0 & t & t & 0 \\
t & \Delta & 0 & t \\
t & 0 & \Delta & t \\
0 & t & t & 2 \Delta
\end{array}\right),
$$

where the four basis states in the configuration space are taken in the order: $|p \uparrow, p \downarrow\rangle,\left|d_{1} \uparrow, p \downarrow\right\rangle,\left|p \uparrow, d_{2} \downarrow\right\rangle$, and $\left|d_{1} \uparrow, d_{2} \downarrow\right\rangle$, with $d_{1}, d_{2}$ denoting the $z^{2}-1$ orbitals on the two Mn sites. Diagonalizing the Hamiltonian matrix, we find the AF ground-state energy to be

$$
E_{\uparrow \downarrow}=\Delta-\sqrt{\Delta^{2}+4 t^{2}}
$$

The exchange energy is obtained by taking the difference between the two ground-state energies Eqs. (4) and (6):

$$
J_{\text {intra }}=\epsilon-\left(\Delta+\sqrt{\epsilon^{2}+4 t^{2}}\right) / 2,
$$

where $\epsilon \equiv \sqrt{\Delta^{2}+4 t^{2}}$ and $J_{\text {intra }}$ stands for $J$ or $J^{\prime}$. Note that the expression for $J_{\text {intra }}$ is always positive indicating an antiferromagnetic interaction irrespective of the magnitude of the electronic parameters.

Bent $\mathrm{Mn}-\mathrm{O}-\mathrm{Mn}$ bond. Even though in the present compound, we have a more or less straight bond, it may deviate markedly from a linear bond for other members of the series. In view of this, we generalize the results of the previous subsection for the case of a bent bond. Taking the Mn-O-Mn bond angle to be $\theta$, we proceed in a similar manner as before.

For the bent bond, one more $\mathrm{O}(p)$ orbital in addition to the $\mathrm{O}\left(p_{z}\right)$ orbital gets involved, making the configuration space somewhat larger than for the straight bond (six dimensional for the FM case and nine dimensional for the AF case). However, the two Hamiltonians can still be diagonalized analytically. The result for the exchange energy is

$$
J_{\text {intra }}=\epsilon-\left[\epsilon^{2}+\sqrt{\epsilon^{4}-16 t^{4} \cos ^{2} \theta}\right]^{1 / 2} / \sqrt{2},
$$

which is again always positive (antiferromagnetic) irrespective of the parameters. In the limit of the straight bond ( $\theta$ $=\pi$ ), this expression can be shown to be equivalent to Eq. (7) after some algebra. Taking the limit $t / \Delta \ll 1$ in the above equation, we obtain the result of the fourth-order perturbation theory:

$$
J_{\text {intra }}=2 t^{4} / \Delta^{3} \times \cos ^{2} \theta .
$$

The above thus explains the antiferromagnetic sign of both $J$ and $J^{\prime}$ consistent with the experiments. ${ }^{8}$

To the best of our knowledge the magnitudes of $J$ and $J^{\prime}$ for $\mathrm{Sr}_{3} \mathrm{Mn}_{2} \mathrm{O}_{7}$ are not known experimentally. However, spinwave measurements for a related compound in the Ruddlesden-Popper series with $40 \%$ La doping indicates $J^{\prime}$ to be substantially lower than $J\left(J \approx 9 \mathrm{meV}\right.$ and $J^{\prime}$ $\approx 3 \mathrm{meV}) .{ }^{18}$ We expect a similar scenario for the present compound based on the differences of the $\mathrm{Mn}-\mathrm{O}$ bond lengths, since the $p d$ tight-binding matrix elements vary as $d^{-7 / 2}, d$ being the distance between the two atoms. ${ }^{19}$ The reduced value of $J^{\prime}$ compared to $J$ is consistent with a longer

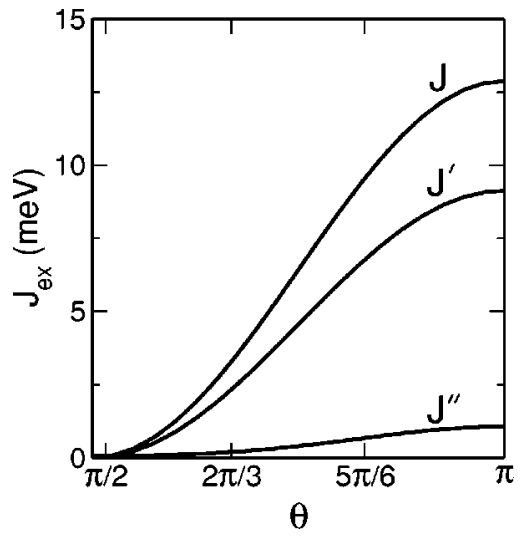

FIG. 8. Intralayer and intrabilayer exchange interactions $J$ and $J^{\prime}$ calculated from Eq. (8) and interbilayer exchange $J^{\prime \prime}$ calculated using Eqs. (2), (11), and (13). The magnitude of $J^{\prime \prime}$ is considerably less as compared to the intralayer exchanges since the former involves a longer superexchange path with four atoms. Parameters are: $\Delta=5 \mathrm{eV}, t_{p d \sigma}=-1 \mathrm{eV}, t_{p p \sigma}=1 \mathrm{eV}$, and $t_{p p \pi}=-0.25 \mathrm{eV}$.

$\mathrm{Mn}-\mathrm{O}(1)$ bond $(1.947 \AA)$ than $\mathrm{Mn}-\mathrm{O}(3)$ (1.895 $\AA$ ) via which the two exchanges are respectively mediated.

The calculated values of $J$ and $J^{\prime}$ as a function of the bond angle $\theta$ are shown in Fig. 8. For the set of parameters $\Delta=5 \mathrm{eV}, \quad t=-1 \mathrm{eV}$, and $\theta \approx 180^{\circ}$, we find $J$ $=12.9 \mathrm{meV}$ and $J^{\prime}=9.1 \mathrm{meV}$. In the calculation of $J^{\prime}, t$ was reduced according to the $d^{-7 / 2}$ scaling mentioned above, while all other parameters were taken to be the same.

\section{B. Interbilayer exchange: $\mathrm{Mn}-\mathrm{O}-\mathrm{O}-\mathrm{Mn}$ case}

We now turn to the interbilayer exchange $J^{\prime \prime}$, which is between two adjacent $\mathrm{Mn}$ atoms located on two different bilayers as shown in Fig. 1. Since the superexchange path is now longer, consisting of four atoms, we expect the exchange to be much weaker. In fact now the exchange interaction occurs only in the sixth order of the perturbation theory, as opposed to the fourth order in the previous case. Experiments indeed indicate the interbilayer coupling to be about two orders of magnitude smaller than the intralayer coupling. ${ }^{18}$

We assume that all four atoms in the superexchange path are in the same plane, which is a good approximation for $\mathrm{Sr}_{3} \mathrm{Mn}_{2} \mathrm{O}_{7}$, and neglect all hoppings that are not crucial to the exchange, retaining only the $p_{z}-p_{z}$ and $d_{3 z^{2}-1}-p_{z}$ hopping channels. For the FM orientation of the two Mn $t_{2 g}$ spins, the Hamiltonian in the six-dimensional $\left({ }^{4} C_{2} \times{ }^{2} C_{2}\right)$ configuration space is given by

$$
H_{\uparrow \uparrow}=\left(\begin{array}{cccccc}
0 & 0 & t & t & 0 & 0 \\
0 & \Delta & 0 & t^{\prime} & 0 & 0 \\
t & 0 & \Delta & 0 & t^{\prime} & -t \\
t & t^{\prime} & 0 & \Delta & 0 & -t \\
0 & 0 & t^{\prime} & 0 & \Delta & 0 \\
0 & 0 & -t & -t & 0 & 2 \Delta
\end{array}\right),
$$


where we have taken the Mn-O hopping $t \equiv t_{z, 3 z^{2}-1}=t_{p d \sigma}$ and the O-O hopping $t^{\prime} \equiv t_{z, z}=t_{p p \sigma} \cos ^{2} \theta+t_{p p \pi} \pi^{\sin ^{2}} \theta, \theta$ being the Mn-O-O angle shown in Fig. 7. The ground-state energy is

$$
E_{\uparrow \uparrow}=\Delta-\left[\Delta^{2}+\tau^{2}+\sqrt{16 \Delta^{2} t^{2}+\left(\Delta^{2}-\tau^{2}\right)^{2}}\right]^{1 / 2} / \sqrt{2},
$$

where $\tau=\sqrt{4 t^{2}+t^{\prime 2}}$. For the AF case, the Hamiltonian is given by

$$
\left.\begin{array}{l}
H_{\uparrow \downarrow} \\
=
\end{array} \begin{array}{ccccccccc}
0 & -t & 0 & 0 & 0 & 0 & -t & 0 & 0 \\
-t & \Delta & t^{\prime} & 0 & 0 & 0 & 0 & -t & 0 \\
0 & t^{\prime} & \Delta & 0 & 0 & 0 & 0 & 0 & -t \\
0 & 0 & 0 & \Delta & -t & 0 & t^{\prime} & 0 & 0 \\
0 & 0 & 0 & -t & 2 \Delta & t^{\prime} & 0 & t^{\prime} & 0 \\
0 & 0 & 0 & 0 & t^{\prime} & 2 \Delta & 0 & 0 & t^{\prime} \\
-t & 0 & 0 & t^{\prime} & 0 & 0 & \Delta & -t & 0 \\
0 & -t & 0 & 0 & t^{\prime} & 0 & -t & 2 \Delta & t^{\prime} \\
0 & 0 & -t & 0 & 0 & t^{\prime} & 0 & t^{\prime} & 2 \Delta
\end{array}\right) .
$$

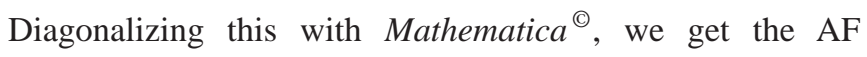
ground-state energy:

$$
E_{\uparrow \downarrow}=4 / 3 \times\left\{\Delta-\alpha \cos \left[\sqrt{4 \alpha^{6}-\beta^{6}} /\left(3 \beta^{3}\right)\right]\right\},
$$

where $\alpha^{2} \equiv \Delta^{2}+3\left(t^{2}+t^{\prime 2}\right)$ and $\beta^{3} \equiv 2 \Delta^{3}+9 \Delta\left(t^{2}-2 t t^{2}\right)$. From numerical diagonalization, we have verified that this expression is valid for typical values of the Hamiltonian parameters. An eigenvalue with a different analytical expression may become the lowest eigenvalue for very different parameters.

The interbilayer exchange $J^{\prime \prime}$ is the difference of the two ground-state energies as before. In the limit of $t, t^{\prime} \ll \Delta$ and taking $t=t^{\prime}$ for the sake of argument, $J^{\prime \prime}$ is given to the lowest order in hopping by the expression

$$
J^{\prime \prime}=E_{\uparrow \uparrow}-E_{\uparrow \downarrow} \sim t^{6} / \Delta^{5},
$$

which is a much weaker exchange as compared to the intralayer exchanges.

Unlike the case of the intralayer exchange, there is no contribution to the interbilayer exchange in the fourth-order perturbation theory. This is easily understood since there is no fourth-order process that would take an electron from one $\operatorname{Mn}\left(3 z^{2}-1\right)$ orbital to the other and back. Therefore the in- terbilayer exchange interaction only includes contributions in energy that are at least of the sixth order in the perturbation theory.

In Fig. 8, we show the calculated values of the exchange $J^{\prime \prime}$ as a function of the Mn-O-O bond angle $\theta$ from diagonalization of the Hamiltonians (10) and (12). Note that for $\mathrm{Sr}_{3} \mathrm{Mn}_{2} \mathrm{O}_{7}$, angle $\theta$ is in the vicinity of $135^{\circ}$, so that $J^{\prime \prime}$ is substantially diminished from the straight-bond result. The weak magnitude of $J^{\prime \prime}$ is borne out by experiments, where the measured value of $J^{\prime \prime}$ is as small as $0.06 \mathrm{meV}^{18}$

Now the crystal structure is such that the antiferromagnetic exchanges $J, J^{\prime}$, and $J^{\prime \prime}$ cannot all be accommodated at the same time leading to spin frustration. Since the intrabilayer exchanges $J$ and $J^{\prime}$ are much stronger compared to the interbilayer exchange $J^{\prime \prime}$, the $\mathrm{Mn}$ moments assume an $\mathrm{AF}$ ordering within the bilayer, while as seen from Fig. 1 only half the Mn-Mn ordering between two Mn atoms belonging to neighboring bilayers are AF, the other half being ferromagnetic. Spin frustration can be removed by making the two equivalent $\mathrm{Mn}-\mathrm{O}(2)-\mathrm{O}(2)-\mathrm{Mn}$ interbilayer superexchange paths nonequivalent, by either a small structural change affecting the $\mathrm{Mn}-\mathrm{O}$ distances or by the presence of electron hopping beyond the nearest neighbor. The observed magnetic structure is therefore consistent with a weak $J^{\prime \prime}$ as found from our results.

\section{SUMMARY}

In summary, we have studied the electronic structure of the bilayer perovskite $\mathrm{Sr}_{3} \mathrm{Mn}_{2} \mathrm{O}_{7}$ from density-functional calculations. The band structure indicates a large mass anisotropy for both the conduction as well as for the valence bands, leading to a strong anisotropy in resistivity for samples with doped carriers within the relaxation-time approximation. We have also found a large resistivity anisotropy in the ferromagnetic metal $\mathrm{LaSr}_{2} \mathrm{Mn}_{2} \mathrm{O}_{7}$ from band calculations. The calculated anisotropy there, $\rho_{c} / \rho_{a b} \sim 40$, is in qualitative agreement with the experimental value of $\sim 100$.

The magnetic structure in $\mathrm{Sr}_{3} \mathrm{Mn}_{2} \mathrm{O}_{7}$ is consistent with the antiferromagnetic superexchange, with the interbilayer exchange being much weaker as compared to the intrabilayer exchange. The electronic structure is similar to that of the perovskite $\mathrm{CaMnO}_{3}$, although the magnetic structure of $\mathrm{Sr}_{3} \mathrm{Mn}_{2} \mathrm{O}_{7}$ is much richer.

\section{ACKNOWLEDGMENTS}

This work was supported by the U. S. Department of Energy under Contract No. DOE FG02-00E0045818.
*Permanent address: Institute for Nuclear Sciences-"Vinča," P.O. Box: 522, 11001 Belgrade, Yugoslavia.

${ }^{1}$ Y. Moritomo, A. Asamitsu, H. Kuwahara, and Y. Tokura, Nature (London) 380, 141 (1996).

${ }^{2}$ T. Kimura, Y. Tomioka, H. Kuwahara, A. Asamitsu, M. Tamura, and Y. Tokura, Science 274, 1698 (1996).

${ }^{3}$ S.N. Ruddlesden and P. Popper, Acta Crystallogr. 11, 54 (1958).
${ }^{4}$ R. Osborn, S. Rosenkranz, D.N. Argyriou, L. Vasiliu-Doloc, J.W. Lynn, S.K. Sinha, J.F. Mitchell, K.E. Gray, and S.D. Bader, Phys. Rev. Lett. 81, 3964 (1998).

${ }^{5}$ L. Onsager, Phys. Rev. 65, 117 (1944).

${ }^{6}$ P.K. de Boer and R.A. de Groot, Phys. Rev. B 60, 10758 (1999).

${ }^{7}$ D. S. Dessau, T. Saitoh, C.-H. Park, Z.-X Shen, P. Villella, N.

Hamada, Y. Moritomo, and Y. Tokura, Phys. Rev. Lett. 81, 192 
(1998)

${ }^{8}$ J.F. Mitchell, J.E. Millburn, M. Medarde, S. Short, J.D. Jorgensen, and M. T. Fernáandez-Díaz, J. Solid State Chem. 141, 599 (1998); J.F. Mitchell, J. Appl. Phys. 85, 4352 (1999).

${ }^{9}$ D. Louca, G.H. Kwei, and J.F. Mitchell, Phys. Rev. Lett. 80, 3811 (1998)

${ }^{10}$ O.K. Andersen, Phys. Rev. B 12, 3060 (1975); H. L. Skriver, The LMTO method (Springer, New York, 1983); O.K. Andersen and O. Jepsen, Phys. Rev. Lett. 53, 2571 (1984).

${ }^{11}$ U. von Barth; and L. Hedin, J. Phys. C 5, 1629 (1972).

${ }^{12}$ S. Satpathy, Z.S. Popović, and F. Vukajlović, J. Appl. Phys. 79, 4555 (1996).

${ }^{13}$ W.E. Pickett and D.J. Singh, Phys. Rev. B 53, 1146 (1996).
${ }^{14} \mathrm{~J}$. M. Ziman, Principles of the Theory of Solids (Cambridge University Press, Cambridge, 1972).

${ }^{15}$ T. Kimura, R. Kumai, Y. Tokura, J.Q. Li, and Y. Matsui, Phys. Rev. B 58, 11081 (1998).

${ }^{16}$ S.Yu. Savrasov and D.Yu. Savrasov, Phys. Rev. B 46, 12181 (1992).

${ }^{17}$ J.P. Perdew and Y. Wang, Phys. Rev. 45, 13244 (1992); J.P. Perdew, J.A. Chevary, S.H. Vosko, K.A. Jackson, M.R. Pederson, D.J. Singh, and C. Fiolhais, ibid. 46, 6671 (1992).

${ }^{18}$ J. F. Mitchell (private communication).

${ }^{19}$ W. A. Harrison, in Electronic Structure and the Properties of Solids (Freeman, San Francisco, 1980). 